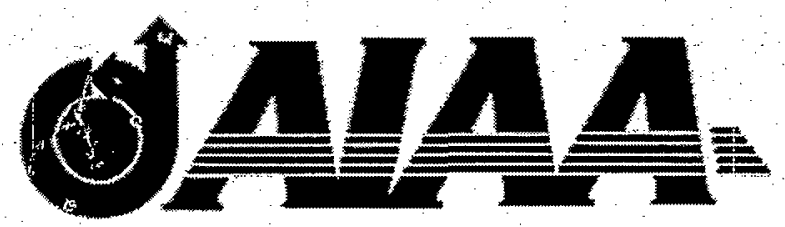

AIAA-99-2576

Sensitivity of Hollow Cathode Performance to Design and Operating Parameters

I. Katz, M.J. Mandell

Maxwell Technologies Systems Division

M. Patterson

NASA/GIenn Research Center

M. Domonkos

Plasmadynamics and Electric

Propulsion Laboratory

University of Michigan

35th AIAA/ASME/SAE/ASEE Joint Propulsion Conference \& Exhibit

June 20-23,1999 / Los Angeles, CA

For permission to copy or republish, contact the American Institute of Aeronautics and Astronautics 370 L'Enfant Prmenade, S.W., Washington, D.C. 20024 


\title{
SENSITIVITY OF HOLLOW CATHODE PERFORMANCE TO DESIGN AND OPERATING PARAMETERS
}

\author{
I. Katz, M. J. Mandell ${ }^{*}$ \\ Maxwell Technologies Systems Division \\ M. Patterson ${ }^{\dagger}$ \\ NASA/Glenn Research Center \\ M. Domonkos † \\ Plasmadynamics and Electric Propulsion Laboratory \\ The University of Michigan
}

An improved orifice ionization model of hollow cathode plasma generation (Katz, Gardner, Mandell, Jongeward, Patterson, Myers, Model of Plasma Contactor Performance, JSR, 34, 1997, p 824) has been applied to a new series of small hollow cathodes being developed at NASA/GRC. A study was made of the sensitivity of the spot mode emission current and voltage as a function of (1) Orifice diameter (2) Orifice length (3) Gas flow rate (4) Keeper current. The results show that the spot mode emission current is maximized at small orifice diameter and high keeper current. Iaboratory measurements confirm the predicted trends, and lead to operating points on existing cathodes where the cathode emits $30 \%$ more electron current while using $30 \%$ less power at a $15 \%$ lower xenon flow rate.

In the study the hollow cathode orifice length, the orifice area, the xenon gas flow, and the keeper current were each changed by a factor of two. The emission current increased faster than linearly with the change in anode current. This results from the power dissipated in the orifice increasing with the square of the current; and thus the ionization also increasing faster than linearly with current. The smaller the orifice diameter, the more effective the ionization, and thus the larger the current that the plasma can support. The increased ionization results from the increase in electrical resistance in a smaller orifice, and thus increased power dissipation.

\section{Nomenclature}

$F \quad=$ gas flow rate, standard cubic centimeter per minute, $\mathrm{sccm}$

$I_{D} \quad=$ total orifice electron current, $\mathrm{A}$

$I_{\text {emission }}=$ orifice current emilled, $\mathrm{A}$

$I_{\text {keeper }}=$ keeper electrode current, A

$I_{\text {loss }} \quad=$ ion loss rate, $\mathrm{A}$

$I_{\max }=$ maximum possible electron current, $\mathrm{A}$

$I_{\text {piod }}=$ total ion production rate, A

$\mathrm{J}_{\mathrm{e}} \quad$ = electron current density, $\mathrm{A} \mathrm{m}^{-2}$

$\mathrm{J}_{\mathrm{i}} \quad=$ ion current density, $\mathrm{A} \mathrm{m}^{-2}$

$L \quad=$ orifice length, $m$

$m \quad=$ neutral atom mass, $\mathrm{kg}$

$m_{e}:=$ electron mass, $\mathrm{kg}$

$$
\begin{array}{ll}
m_{i} & =\text { ion mass, } \mathrm{kg} \\
m_{n} & =\text { neutral atom mass, } \mathrm{kg} \\
n & =\text { neutral atom density mass, } \mathrm{m}^{-3}
\end{array}
$$

$n_{e} \quad=$ electron density, $\mathrm{m}^{-3}$

$n_{i} \quad=$ ion density, $\mathrm{m}^{-3}$

$N_{n} \quad=$ neutral density; $\mathrm{m}^{-3}$

$P \quad$ = Power dissipated in the orifice, $\mathrm{W}$

$R \quad=$ electrical resistance of the orifice, ohms

$r \quad=$ orifice radius, $\mathrm{m}$

$W_{\text {conv }}=$ power loss by electron convection, $\mathrm{W}$

$W_{\text {ion }}=$ power loss by ionization, $\mathrm{W}$

$W_{\mathrm{rad}} \quad=$ power loss by radiation, $\mathrm{W}$

$\varepsilon_{o} . \quad=$ permeability constant, $\mathrm{Fm}^{-1}$.

* 8888 Balboa Ave., San Diego, CA 92123-1506.

$\dagger 21000$ Brookpark Rd., Cleveland, OH 44135.

$\div$ Ann Arbor, MI 48109 .

Copyright (-) 1999 by the American Institute of

Aeronautics and Astronautics, Inc. All rights reserved. 


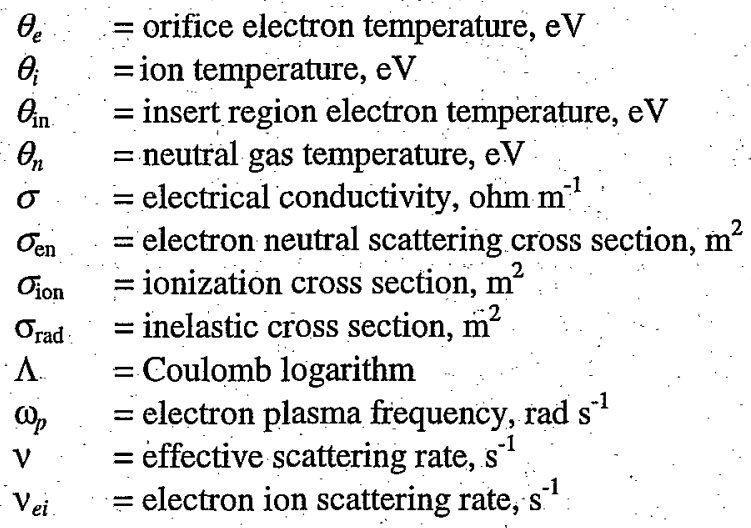

\section{Introduction}

The push to smaller spacecraft has led to a corresponding effort to develop small, low power electric propulsion systems which retain the overall high efficiency of larger systems. Hollow cathodes (HC) are a major component in both electrostatic ion thrusters and Hall effect thrusters. The work presented below is part of an effort by the On-Board Propulsion Branch of the NASA Glenn Research Center to develop a new class of hollow cathode that is half the diameter of the conventional design. One of the new, smaller, cathodes is shown in Figure 1 along with a conventional hollow cathode.

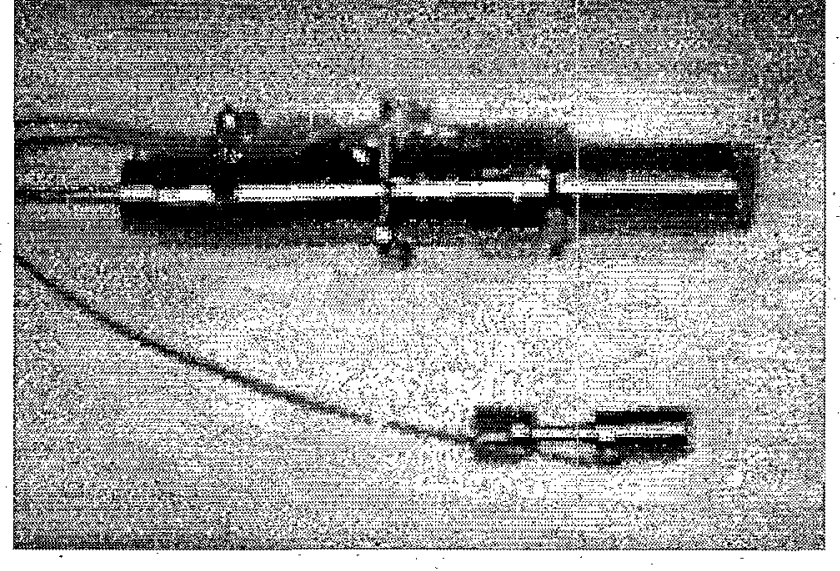

Fig. 1. A new, smaller, hollow cathode (hottom) compared with the ISS Plasma Contactor.

Hollow cathodes (HC's) are devices that emit copious electron currents through a self generated plasma. The expellant is typically an inert gas (xenon or argon), but liquid metals have also been used. These devices typically operate at low voltages (10-30V), and emit an ampere or more of electron current for an expellant flow rate substantially below an ampere equivalent. When enough plasma is generated so that no anode sheath develops, the cathode is said to be operating in spot mode, named for the small bright spot at the cathode orifice. When the current drawn exceeds the limit that the cathode plasma can supply, the cathode transitions to plume mode, named after the large glowing plume exterior to the cathode. Plume mode also involves higher voltages, and is a less efficient mode of operation.

To aid in the development of these new hollow cathodes, an improved orifice ionization model of hollow cathode plasma generation ${ }^{1}$ has been used to study the sensitivity of the spot mode emission current and voltage as a function of $\mathrm{HC}$ design and operating parameters, including

1. Orifice diameter

2. Orifice length

3. Gas flow rate

4. Keeper current.

The goal was to reduce the design space, and to see if the model was accurate enough to be a useful design tool. The model and the results are presented below.

\section{Review of HC Orifice Ionization Model}

Figure 1 shows the typical dimensions of a hollow cathode for space applications. Though the entire device is fairly small, the orifice through which the insert region communicates with the external space is an order of magnitude smaller still. As this region has an extremely high current density, processes taking place in the orifice region govern the amount of plasma flowing from the device. Typically, the orifice length is much greater than the orifice diameter. This means that only a tiny fraction of the ions entering the orifice can exit the orifice without hitting the orifice wall and recombining. As we show below, almost all the ions that exit were generated in the orifice.

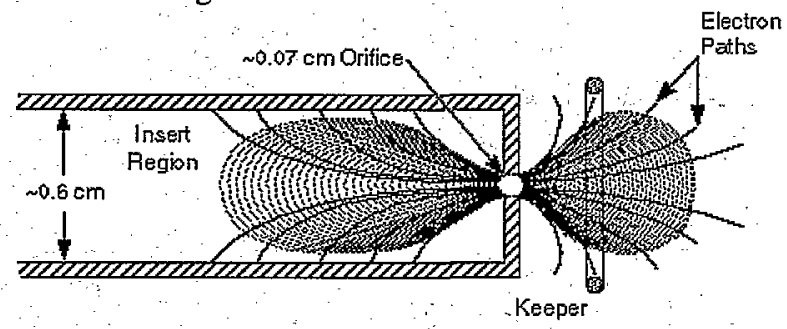

Fig. 2. Typical dimensions of a conventional hollow cathode for space applications. 


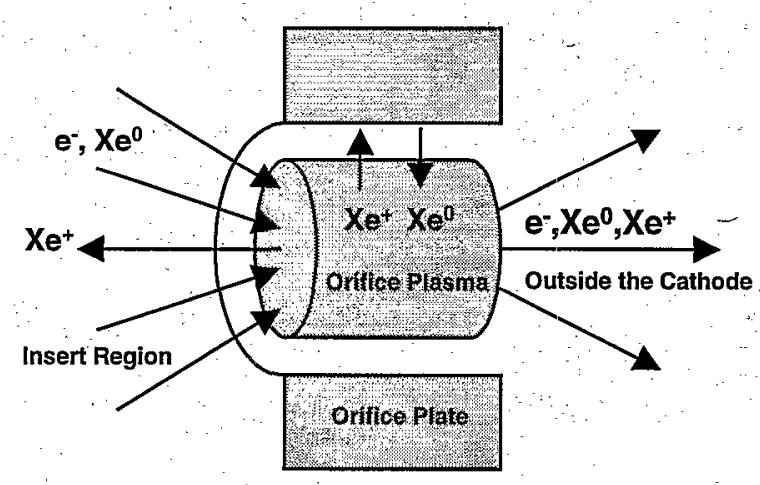

Fig. 3. Magnified view of the cylindrical orifice region.

Operation of hollow cathodes has been investigated in the laboratory by Siegfried and Wilbur ${ }^{2}$. Previous studies (e.g., References 3 and 4) have analyzed the physical processes inside the hollow cathode which lead to electron emission from the insert. In this paper we present an improved model of the ionization and energy balance in the orifice of a hollow cathode. The model is consistent with previous models of the physics interior to the hollow cathode, but investigates in more detail what occurs within the tiny orifice region.

\section{Model of Urifice Processes}

The orifice is so small that, although it is many times larger than a plasma debye length, it extends for only a few mean free paths for electrons, ions, or neutrals. It is therefore reasonable to treat the orifice as containing a homogeneous neutral plasma with a thin wall sheath. Known are the gas flow through the device and the discharge current. We calculate the electron temperature and plasma density in the orifice, the plasma output of the device, the voltage required to sustain the discharge current through the orifice, and the energy budget of the orifice region. The model now includes energy dependent electron neutral scattering cross section based on literature values.

\section{Electron Temperature}

In order to have a steady state plasma in the orifice, the electrons must be sufficiently hot to create ions at a rate equal to the loss of ions to the orifice wall or by flow out the ends. We assume that:

(1) The neutral gas density, $N_{n}$, is known.

(2) The electron energy distribution is maxwellian with temperature $\theta_{\mathrm{c}}$.
(3) The ions are accelerated toward the orifice boundaries by quasi-neutral electric fields. We assume $\theta_{i}=0.1 \mathrm{eV}$.

(4) The plasma is quasi-neutral, so that $\mathrm{n}_{\mathrm{i}}=\mathrm{n}_{\mathrm{e}}=\mathrm{n}$.

For a cylindrical orifice of radius $r$ and length $L$, the ion production rate (amperes) is given by

$$
\mathrm{I}_{\text {prod }}=\pi \mathrm{r}^{2} \mathrm{~L} \times 4 \sigma\left(\theta_{\mathrm{e}}\right) \times \mathrm{J}_{\mathrm{e}} \mathrm{N}_{\mathrm{n}}
$$

$\sigma\left(\theta_{\mathrm{e}}\right)$ is the thermally averaged cross-section for electron impact ionization of Xenon ${ }^{5}$, which, for temperatures up to several $\mathrm{eV}$ is well fit by

$$
\begin{aligned}
& \sigma\left(\theta_{\mathrm{e}}\right)=\left[3.97+0.643 \theta_{\mathrm{e}}-0.0368 \theta_{\mathrm{e}}^{2}\right] \times \\
& \mathrm{e}^{-12.127 / \theta_{\mathrm{e}} \times 10^{-20} \mathrm{~m}^{2}}
\end{aligned}
$$

$\mathrm{J}_{\mathrm{e}, \mathrm{i}}$ is the electron or ion thermal current

$$
\mathrm{J}_{\mathrm{e}, \mathrm{i}}=\mathrm{ne}\left(\mathrm{e} \theta_{\mathrm{e}, \mathrm{i}} / 2 \pi \mathrm{m}_{\mathrm{e}, \mathrm{i}}\right)^{1 / 2}
$$

The neutral density is calculated by assuming the flow rate equals the thermal efflux through the orifice cross-sectional area:

$$
\pi \mathrm{r}^{2} \times \mathrm{eN}_{\mathrm{n}}\left(\mathrm{e} \theta_{\mathrm{n}} / 2 \pi \mathrm{m}_{\mathrm{n}}\right)^{1 / 2}=\left(0.0718 \mathrm{~F}-\mathrm{I}_{\mathrm{i}}\right)
$$

where $F$ is the gas flow rate (sccm), and $I_{i}$ is the ion output of the device (amperes), and the neutral temperature; $\theta_{\mathrm{n}}=0.1 \mathrm{eV}$. The ion loss rate is given by

$$
\mathrm{I}_{\mathrm{loss}}=2 \pi \mathrm{r}(\mathrm{r}+\mathrm{L}) \mathrm{J}_{\mathbf{i}}
$$

The steady state condition is that the ion production equals the ion losses.

$$
\mathrm{I}_{\text {prod }}=\mathrm{I}_{\text {loss }}
$$

If we neglect ion production compared with gas flow, the electron temperature is independent of plasma density and depends weakly on the gas flow rate and the ion and neutral temperatures. The electron temperature in the orifice is about $2 \mathrm{eV}$ for all low-utilization hollow cathodes.

\section{Electrical Resistance}

Plasma conductivity; $\sigma$, is given by 


$$
\begin{aligned}
& \sigma=\varepsilon_{0} \omega_{\mathrm{p}}^{2} / v \\
& \omega_{\mathrm{p}}^{2}=\mathrm{n}^{2} / \varepsilon_{0} \mathrm{~m}_{\mathrm{e}}
\end{aligned}
$$

where $\omega_{\mathrm{p}}$ is the electron plasma frequency, and $v$ is the effective scattering rate for conduction. The scattering rate, $v$, is the sum of

(1) Electron-ion scattering:

$$
v_{\mathrm{ei}}=2.9 \times 10^{-12} \mathrm{n}_{\mathrm{e}} \Lambda \theta_{\mathrm{e}}^{-3 / 2}
$$

and

(2) Electron-neutral scattering:

$$
\begin{aligned}
& \sigma_{\text {en }}=6.6 \times 10^{-19}(\theta / 4-0.1) /\left(1+(\theta / 4)^{1.6}\right) \\
& \nu_{\mathrm{en}}=\sigma_{\mathrm{en}} \mathrm{N}_{\mathrm{n}}(\mathrm{e} \theta / \mathrm{m})^{1 / 2}
\end{aligned}
$$

where the neutral cross section was found by integrating the energy dependent total electron cross section from Wagcnaar and Heer over a Maxwellian distribution. This formula is good for electron temperatures between $1 \mathrm{and} 10 \mathrm{eV}$.

The quantity $\Lambda$, the coulomb logarithm, given by

$$
\Lambda=30-1 / 2 \ln \left(\mathrm{n}_{\mathrm{e}} \theta_{\mathrm{e}}^{-3}\right)
$$

is about 7 for the orifice conditions. We neglect any non-classical scattering. The electrical resistance, $R$, of the orifice is then

$$
\mathrm{R}=\mathrm{L} / \pi \mathrm{r}^{2} \sigma
$$

and the voltage across the orifice $\left(\mathrm{V}=\mathrm{I}_{D} \mathrm{R}\right)$ and the power dissipated in the orifice $P=I_{D}^{2} R$ are related to the discharge current by Ohm's Law.

\section{Energy Loss Mechanisms}

There are three major energy loss mechanisms
(6) for the electron gas in the orifice region. Excited or ionized Xenon atoms either radiate to the external world; leave the orifice, or are quenched at the walls, so that these processes are major loss mechanisms. Another significant loss is by electron convection, which is simply a statement that the insert electron temperature is cooler than the orifice electron temperature. The expressions for these losses are:

(1) Ionization loss:

$$
\mathrm{W}_{\text {ion }}=<\mathrm{E}_{\text {ion }}>\times \mathrm{I}_{\text {prod }}
$$

where $\left\langle\mathrm{E}_{\text {ion }}>\right.$ is the mean energy loss due to an ionization event (taken to be $12.2 \mathrm{eV}$ for Xenon).

(2) Radiation loss:

$$
\mathrm{W}_{\mathrm{rad}}=\pi \mathrm{r}^{2} \mathrm{~L} \times<\mathrm{E}_{\mathrm{rad}}>\times 4 \sigma_{\mathrm{rad}}\left(\theta_{\mathrm{e}}\right) \times \mathrm{J}_{\mathrm{e}} \mathrm{N}_{\mathrm{n}}
$$

where $\left\langle\mathrm{E}_{\mathrm{rad}}>\right.$ is the mean energy loss due to an excitation event (taken to be $10 \mathrm{eV}$ for Xenon), and $\sigma_{\text {rad }}$ $\left(\theta_{\mathrm{e}}\right)$, the thermally averaged cross-section for radiative excitation ${ }^{5}$, is approximated for Xenon for temperatures of a few eV by

$$
\sigma_{\text {rad }}\left(\theta_{\mathrm{e}}\right) \sim 1.93 \times 10^{-19} \theta_{\mathrm{e}}^{-1 / 2} \mathrm{e}^{-11.6 / \theta_{\mathrm{e}}}
$$

(3) Convective loss:

$$
\mathrm{W}_{\text {conv }}=\mathrm{I}_{\mathrm{D}}\left(\theta_{\mathrm{e}}-\theta_{\text {in }}\right)
$$

where $I_{D}$ is the discharge current (i.e., the electron current through the orifice), and $\theta_{\text {in }}$ is the electron temperature at the input to the orifice.

The orifice plasma density is found by balancing the ohmic heating with the sum of the three energy losses:

$$
I_{D}^{2}=W_{\text {ion }}+W_{\text {rad }}+W_{\text {conv }}
$$

Table 1. HC orifce dimensions operation parameters and calculated results varying the model parameters.

$\begin{array}{cccccccccc}\text { Diameter } & \text { Length } & \text { Janode }(\mathrm{A}) & \mathrm{Xe}(\mathrm{sccm}) & \text { Ie } \max (\mathrm{A}) & \mathrm{IR}(\mathrm{V}) & \mathrm{I}^{2} \mathrm{R}(\mathrm{W}) & \text { Utilization } & \text { eV/ion } \\ 1.0 & 6.0 & 1.00 & 2.00 & 1.11 & 22.31 & 22.31 & 4 \% & 3,952 \\ 1.0 & 12.0 & 1.00 & 2.00 & 1.18 & 44.26 & 44.26 & 4 \% & 7,315 \\ 0.7 & 6.0 & 1.00 & 2.00 & 1.49 & 40.44 & 40.44 & 5 \% & 5,312 \\ 1.0 & 6.0 & 1.00 & 4.00 & 1.15 & 25.10 & 25.10 & 2 \% & 4,270 \\ 1.0 & 6.0 & 2.00 & 2.00 & 4.77 & 35.06 & 7011 & 17 \% & 2,879\end{array}$




\section{$\underline{\text { Results }}$}

The above equations are used in a spreadsheet to calculate self-consistently the plasma density, electron temperature, power dissipation, and ion output $\left(\pi r^{2} \check{J}_{\mathfrak{j}}\right)$ of the orifice as a function of orifice dimensions, gas flow rate, and discharge current. The calculation consists of iterating the orifice plasma temperature and density until the ion production equals the ion loss and the $I^{2} R$ power dissipation in the orifice equals the total energy loss. The maximum electron current $I_{\max }$ which can flow through the orifice is the one sided electron thermal current times ion current ${ }^{7}$

$$
\mathrm{I}_{\mathrm{e}}^{\max }=\mathrm{f}_{\mathrm{esc}} \sqrt{\frac{\mathrm{m}_{\mathrm{i}}}{2 \pi \mathrm{m}_{\mathrm{e}}}} \mathrm{I}_{\text {ion }}
$$

where $f_{\text {esc }}$ is the fraction of ions that leave the orifice and escape through the hole in the keeper anode, typically about $40 \%$ of the ions.

\section{Sensitivity Studies}

We applied the model described to a hollow cathode design being tested in the laboratory to determine how cathode design and operating parameters control cathode performance. The results are shown in Table 1. The nominal model cathode parameters (first row of the table) were chosen to be similar to those of an early small cathode design. The model was then run changing relevant parameters by a factor of two.

In the second row the orifice length was doubled. The ion output was increased a little, but the power into the orifice increased by a factor of two. This follows naturally from Equation 10, where the resistance is linearly dependent on the orifice length: The additional power is dissipated by radiation ionization losses to the increased wall area. A shorter orifice reduces these losses. However, the orifice length can be reduced only so far to improve performance. The simple zero dimensional model breaks down if the orifice length is less than the orifice diameter.

In the third row, the orifice diameter was reduced so that the electron current density in the orifice was doubled. Again from Equation 10, the resistance doubled, and thus the voltage drop in the orifice also doubled. But the ion generation increased, because the power is deposited in a smaller volume. The ion generation increased more slowly than the power dissipation, consequently it cost more energy for each ion generated. Notice that the energy to generate an ion which escapes from the cathode is quite high.
The gas flow rate was doubled in the fourth case. This increased the resistance somewhat, but made little difference on most of the other parameters. Gas flow is an important parameter only when the gas flow is reduced so much that the ionization inside the orifice is high.

In the final row, the current is doubled. Increasing the current increases the voltage drop in the orifice, and increases the power dissipated in the orifice by a factor of four. This leads to a substantial incrcase in ionization.

Below: we identify the relative sensitivity of the calculated results to variation of input parameters. Since the model is not exact, it is of most use a guide to reducing the trade space in the laboratory necessary to develop an optimum design.

Table 2. Relative variation of $\mathrm{HC}$ model performance to the different parameters. Sensitive parameters are shown in bold.

$\begin{array}{llllll} & \mathrm{I}_{\mathrm{e}} \max & \mathrm{IR} & \mathrm{P}_{\text {in }} & \text { Utilization } & \text { eV/ion } \\ \text { baseline } & 1.00 & 1.00 & 1.00 & 1.00 & 1.00 \\ 2 \text { X length } & 1.07 & \mathbf{1 . 9 8} & 1.98 & 1.07 & \mathbf{1 . 8 5} \\ 2 \mathrm{X} \text { Je } & \mathbf{1 . 3 5} & \mathbf{1 . 8 1} & \mathbf{1 . 8 1} & \mathbf{1 . 3 5} & \mathbf{1 . 3 4} \\ 2 \mathrm{X} \text { gas } & 1.04 & \mathbf{1 . 1 3} & 1.13 & \mathbf{0 . 5 2} & 1.08 \\ 2 \text { X current } & \mathbf{4 . 3 1} & 1.57 & \mathbf{3 . 1 4} & \mathbf{4 . 3 1} & \mathbf{0 . 7 3}\end{array}$

Table 2 shows the sensitivity, within the range of parameters with low expellant utilization. Additional orifice length wastes power because a larger fraction of the ions generated are lost to the orifice wall. An ideal cathode would have a very short orifice. Increasing the current density by decreasing the orifice radius results in higher power and neutral densities within the orifice. The limiting factor is probably increased orifice temperature as the current density keeps increasing. The increased cost per ion is result of the orifice aspect ratio changing when the diameter decreased and the length was held constant. Increasing the gas flow only wastes gas, as long as the ionization fraction is low. Increasing the electron current through the orifice increases the power density by a factor of four, and results in increased ionization, expellant utilization, and a reduced cost energy cost per ion.

\section{Application to Cathode Operation}

A few cathodes with differing orifice geometry were being evaluated. On the basis of the model predictions, the cathode with the smallest orifice diameter and length was selected as being potentially more efficient. In Table 3 are shown the calculated parameters for the baseline cathode and the smaller one 
Table 3. Model performance for $0.25 \mathrm{~A}$ emission of the baseline and smaller orifice cathodes.

$\begin{array}{ccccccccc}\text { Diameter } & \text { Length } & \text { Janode }(\mathrm{A}) & \mathrm{Xe}(\mathrm{sccm}) & \mathrm{Ie} \max (\mathrm{A}) & \mathrm{IR}(\mathrm{V}) & \mathrm{I}^{2} \mathrm{R}(\mathrm{W}) & \text { Utilization } & \text { eV/ion } \\ 1.0 & 6.0 & 1.25 & 1.72 & 1.69 & 25.31 & 31.64 & 7 \% & 3,667 \\ 0.6 & 2.0 & 0.73 & 1.46 & 0.77 & 12.60 & 9.20 & 4 \% & 2,344 \\ 0.6 & 2.0 & 0.98 & 1.46 & 1.33 & 15.29 & 14.99 & 6 \% & 2,205 \\ 0.6 & 2.0 & 1.23 & 2.24 & 2.05 & 18.92 & 23.27 & 7 \% & 2,228\end{array}$

with total current composed of $0.25 \mathrm{~A}$ emission current as well as the anode current, which varied from case to case.

Experimental data is shown in Figure 4. The data shows clearly that smaller cathode performs better than the baseline cathode, even more so than the model predicts. The model predicts that the baseline cathode performance should lie between the two upper cathodes. However, the smaller orifice cathode actually out performs the baseline at for lower gas flow and lower anode current.

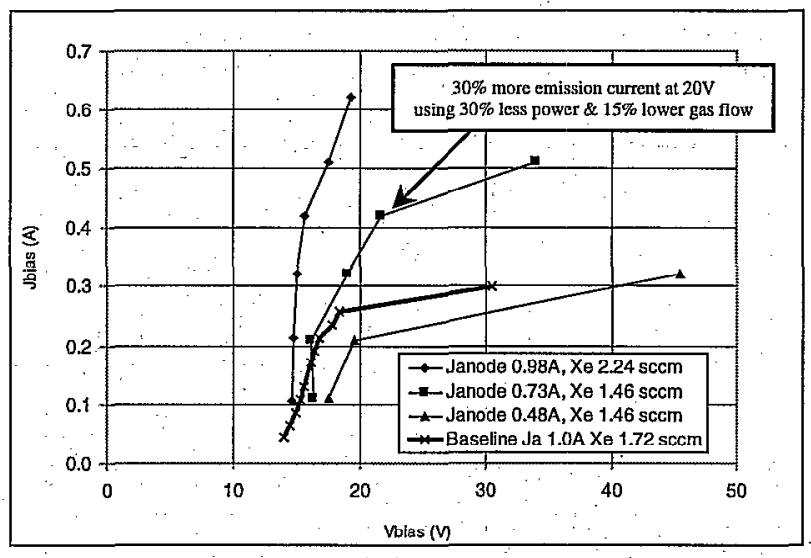

Fig. 4. Laboratory data comparing baseline and small orifice cathode performance

\section{Conclusion}

The model presented shows that the dimensions of the orifice and the total electron current are the most important parameters in determining hollow cathode plasma production. The model provided useful direction for laboratory studies. It was able to correctly rank the performance for a small orifice geometry varying flow rate and anode current, and it did successfully predict that the small orifice cathode would perform better than the baseline cathode. However, it was not sufficiently accurate to predict the relative performance between several operating conditions on different cathodes.

The fundamental information from the model is that the hollow cathode plasma production is controlled by the ion current that is generated in the orifice and escapes without recombining on cathode surfaces.
Fundamental changes in orifice geometry, which allow more of the ions to escape, hold great promise in dramatically improving hollow cathode performance.

\section{References}

1. Katz; I., B. M. Gardner, M. J. Mandell, G. A. Jongeward, M. Patterson, R. M. Myers, J. Spacecraft and Rockets, 34, 1997, p. 824.

2. Siegfried, D.E. and P.J: Wilbur, "An Investigation of Mercury Hollow Cathode Phenomena," AIAA 78-705, AIAA/DGLR 13th International Electric Propulsion Conference, San Diego, 1978.

3. Salhi, A. and P.J. Turchi, "A First-Principles Model for Orificed Hollow Cathode Operation," AIAA 92-3742, 1992.

4. Salhi, A. and P.J. Turchi, "Theoretical Modeling of Orificed, Hollow Cathode Discharges," IEPC-93-024, 1993.

5. Hayashi, Makoto, "Determination of Electron-Xenon Total Excitation Cross-Sections, from Threshold to $100 \mathrm{eV}$, From Experimental Values of Townsend's $\alpha$," J. Phys. D: Appl: Phys., 16, 1983, p. 581.

${ }^{6}$.Wagenaar, R. W. and F: J. de Heer, Total Cross sections for electron scattering from $\mathrm{Ar}, \mathrm{Kr}$, and $\mathrm{Xe}, J$. Phys. B: At. Mol. Phys. 18, 1985, p. 2021.

7. Parks, D. E., Katz; I., Buchholtz, B., and Wilbur, P., "Expansion and Electron Emission Characteristics of a Hollow-Cathode Plasma Contactor," J. Appl. Phys. 74 (12) 1993, p. 7094.

\section{Acknowledgment} 75634-J 